\title{
Author Correction: Biomechanical Stretch Induces Inflammation, Proliferation, and Migration by Activating NFAT5 in Arterial Smooth Muscle Cells
}

\author{
Wei Cao, ${ }^{1,2}$ Donghui Zhang, ${ }^{1,2}$ Qiannan $\mathrm{Li}^{3}{ }^{3}$ Yue Liu, ${ }^{4}$ Shenhong Jing, ${ }^{1,2}$ Jinjin Cui, ${ }^{1,2}$ Wei Xu, ${ }^{1,2}$ \\ Shufeng Li, ${ }^{1,2}$ Jingjin Liu, ${ }^{1,2}$ and Bo $\mathrm{Yu}^{1,2,5}$
}

Author Correction: Inflammation (2017) 40(6):2129-2136

https://doi.org/10.1007/s10753-017-0653-y

After the publication of our article, we became aware that there were errors in Figs. $3 \mathrm{c}$ and d; the figures were incorrectly presented. Our figures in Figs. $3 \mathrm{c}$ and $\mathrm{d}$ are confused with another by the side research project. These errors do not affect the discussion or conclusions in the article. The correct versions of Figs. $3 \mathrm{c}$ and $\mathrm{d}$ are shown.

We apologize to the journal and to the readers for these errors.

Publisher's Note Springer Nature remains neutral with regard to jurisdictional claims in published maps and institutional affiliations.

The online version of the original article can be found at https://oi.org/ 10.1007/s10753-017-0653-y

${ }^{1}$ Department of Cardiology, The Second Affiliated Hospital of Harbin Medical University, Harbin, 150086, People's Republic of China

${ }^{2}$ The Key Laboratory of Myocardial Ischemia, Chinese Ministry of Education, Harbin, 150081, Heilongjiang, People's Republic of China

${ }^{3}$ Department of Geriatric, The Second Affiliated Hospital of Harbin Medical University, Harbin, 150081, Heilongjiang, People's Republic of China

${ }^{4}$ Department of Cardiology, The First Affiliated Hospital of Harbin Medical University, Harbin, 150001, Heilongjiang Province, China

${ }^{5}$ To whom correspondence should be addressed at Department of Cardiology, The Second Affiliated Hospital of Harbin Medical University, Harbin, 150086, People’s Republic of China. E-mail: yubodr@163.com 


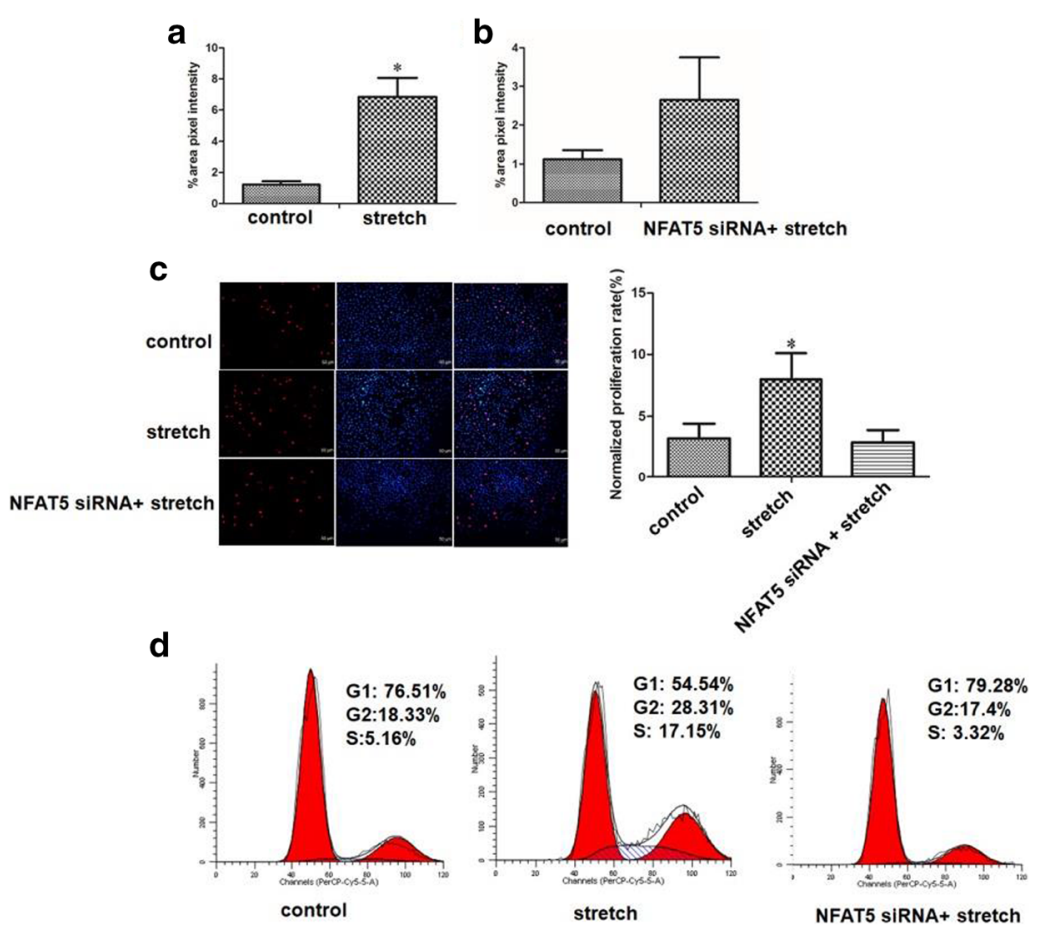

Fig. 3. NFAT5 deficiency impairs HUASMC migration and proliferation. a Biomechanical stretch-stimulated HUASMCs migration. b NFAT5-siRNAtreated HUASMCs migration. $\mathbf{c}$ The cells were stained with EdU and DAPI after transfection with the biomechanical stretch and NFAT5-siRNA treatment. $\mathbf{d}$ Cell cycle distribution of HUASMCs after biomechanical stretch and NFAT5-siRNA treatment. The results are shown as the means \pm SD of at least three separate experiments. $* p<0.05$. 